\title{
DE UM SISTEMA NACIONAL À ADOÇÃO DE PADRÕES INTERNACIONAIS: POR UMA NOVA NORMA BIBLIOGRÁFICA
}

\section{FROM NATIONAL TO INTERNATIONAL STANDARDS: THE ADOPTION OF A NEW BIBLIOGRAPHICAL STYLE}

\author{
Jimena Felipe Beltrão ${ }^{1}$, Taíse da Cruz Silva ${ }^{2}$
}

Recebido em: 23-12- 2018

Aceito em: 31-03-2019

\begin{abstract}
Resumo
Com o objetivo de identificar as normas bibliográficas adotadas por periódicos da área de Ciências Humanas, estudo de caráter quali-quantitativo utilizou pesquisa bibliográfica e levantamentodiagnóstico em revistas indexadas na Scientific Electronic Library Online (SciELO) e na Red de Revistas Científicas de América Latina y el Caribe, España y Portugal (Redalyc). Foram examinados os padrões bibliográficos utilizados em 31 títulos, dos quais nove títulos nacionais e sete internacionais disponíveis na SciELO, além de 15 títulos internacionais indexados na Redalyc. Dentre os quatro padrões identificados, um se destaca pela frequência e pela simplicidade. Com base no diagnóstico, o estudo recomenda o APA, da American Psychological Association, como estilo mais adequado para adoção pelo Boletim do Museu Paraense Emílio Goeldi. Ciências Humanas.
\end{abstract}

Palavras-chave: Periódico científico. Boletim do Museu Paraense Emílio Goeldi. Ciências Humanas. Estilo normativo. Padrão internacional.

\begin{abstract}
A quali-quantitave study was conducted to identify which international bibliographical standards are used among Social Sciences journals indexed in Scientific Electronic Library Online (SciELO) and in Red de Revistas Científicas de América Latina y el Caribe, España y Portugal (Redalyc). By the use of bibliographical research as well as analysis of a total of 31 journals normative standards, research findings indicate four more frequently-used styles in journals in the field. The APA style, from the American Psychological Association, stands out as the more frequent and of most simple format among the remainder and, thus, has been recommended as the bibliographical style to be adopted by the Boletim do Museu Paraense Emílio Goeldi. Ciências Humanas.
\end{abstract}

Keywords: Scientific journal. Boletim do Museu Paraense Emílio Goeldi. Ciências Humanas. Bibliographical style. International standards.

\section{INTRODUÇÃO}

Diante da importância de se dar acesso ao conhecimento científico e da necessidade de agilizar os processos que levam a público resultados de pesquisa, reputa-se que a otimização do tempo e a simplificação das ferramentas de processamento de conteúdo são condições primordiais. Mecanismos de submissão online, de verificação de similaridade e de publicação

\footnotetext{
${ }^{1}$ Ph.D em Ciências Sociais - Analista em Ciência e Tecnologia do Museu Paraense Emílio Goeldi (MPEG), Editora Científica do Boletim do Museu Paraense Emílio Goeldi. Ciências Humanas. e-mail: jimenafelipebeltrao@gmail.com

${ }^{2}$ Graduada em Biblioteconomia - Bolsista do Programa de Capacitação Institucional do MCTI - PCI do Museu Paraense Emílio Goeldi. e-mail: taisesilva@museu-goeldi.br
} 
contínua de trabalhos inéditos sempre baseados em condutas éticas já vêm sendo implementados como parte do processo de produção editorial de artigos científicos.

O nível de exigência com vistas à qualidade dos conteúdos e à confiabilidade dos procedimentos que geram tal conhecimento estão associados, na atualidade, a fortes controles anti-plágio e a uma tendência à internacionalização e à profissionalização de processos editoriais. Todos esses itens compõem um esforço maior da Ciência Aberta, movimento de ampliação do acesso ao conhecimento que deve ser de domínio público. Para Rodrigues (2004, p. 25) isso implica na: “[...] disponibilização livre na Internet de literatura de carácter académico ou científico, permitindo a qualquer utilizador ler, descarregar, copiar, distribuir, imprimir, pesquisa ou referenciar o texto integral dos documentos." Com esse mesmo enfoque, em junho de 2012, a Royal Society já havia publicado no guia "Science as an open enterprise" "[...] open data (available, intelligible, assessable and useable data) combined with open access to scientific publications and effective communication of their contents [...]." (THE ROYAL SOCIETY, 2012, p. 16). Mais recentemente, periódicos científicos que adotam o acesso aberto receberam apoio em artigo intitulado Who's afraid of peer review?, onde o autor diagnostica que "[...] some open-access journals that have been criticized for poor quality control provided the most rigorous peer review of all. [...]" (BOHANNON, 2013, p. 61).

No Plano de Desenvolvimento Editorial ${ }^{1}$ do Boletim do Museu Paraense Emílio Goeldi. Ciências Humanas ${ }^{2}$, a editora da revista Jimena Felipe Beltrão informa sobre o que já é uma tradição no ambiente da ciência pública: "A revista é de acesso aberto, o que significa que todo o conteúdo está disponível gratuitamente, sem custo para o usuário ou instituição, bem como não há taxas para submissão de manuscritos." (BOLETIM DO MUSEU PARAENSE EMÍLIO GOELDI. CIÊNCIAS HUMANAS, 2018, p. 2).

Parte desse cenário, a norma observada pelas revistas científicas deve ser de domínio o mais amplo possível, com padrões internacionais e de fácil aplicação tanto no que se refere à forma como também à geração. Para Stumpf (2008, p. 19) "O que pôde ser observado nos últimos tempos é que houve avanços na qualidade das revistas de comunicação, tanto nos aspectos intrínsecos quanto nos extrínsecos, haja vista a preocupação dos editores em seguir algumas normas de apresentação formal e de conteúdo dos artigos.”

As normas adotadas pelas revistas científicas são importantes, pois como argumentam Medeiros, Fachin e Rados (2008, p. 417): "A normalização é essencial para o aumento do prestígio, reconhecimento e aceitabilidade dos periódicos científicos." Ainda sobre o tema, Santa Anna (2017, p. 62) corrobora que "[...] Normalização Bibliográfica tem sua importância garantida, uma vez que, sem a Normalização, provavelmente, o processo comunicativo seria comprometido." É de especial interesse destacar a diferença entre os conceitos de normatização e normalização. Em Sabadini, Sampaio e Nascimento (2009, p. 38), "Normatização é o ato de criar e construir normas; é a criação de regras. A normalização é a aplicação de norma com o objetivo de ordenar e dar padrão a uma atividade." Essa prática de normalização é executada pelo profissional reconhecido na área. Para Maimone e Tálamo (2008, p. 311) "[...] entre as muitas competências atribuídas ao bibliotecário, três delas que nos chamam a atenção para o tema da editoração: a normalização de documentos; a análise de trabalhos técnicos-científicos e, a organização e gerenciamento de dados virtuais [...]."

O BMPEG. Ciências Humanas atualmente utiliza norma nacional definida pela Associação Brasileira de Normas Técnicas (ABNT): as NBR $6023^{3}$ de elaborações de referências e a NBR 10520 de citações. Em razão da necessidade de utilização de padrão

\footnotetext{
${ }^{1}$ Apresentado ao SciELO em 2018

${ }^{2}$ Doravante BMPEG. Ciências Humanas.

${ }^{3}$ O BMPEG. Ciências Humanas usa a ABNT NBR 6023 (2002). Em 2018, houve a divulgação da NBR 6023 (2018). Mas no momento da produção deste artigo, a revista ainda não fazia uso da nova versão, posto que os três números de 2018 já se encontravam em circulação
} 
internacional e de formato mais simples e de fácil aplicação, procurou-se estudar padrões alternativos aos praticados exclusivamente no Brasil com vistas a uma eventual mudança.

O estudo para propor a mudança da Associação Brasileira de Normas Técnicas (ABNT) de um novo estilo/padrão na elaboração de referências e de citações no BMPEG. Ciências Humanas se justifica porque a ABNT é uma norma eminentemente nacional, complexa e de uso sobretudo por publicações brasileiras. Ainda que se reconheça o mérito de criação de uma normativa nacional, o fato é de que ao não ser de domínio internacional, a aplicação é dificultada entre autores internacionais. A utilização de norma complexa amplia o trabalho e o tempo de verificação, edição das submissões, o que representa demora adicional na publicação de resultados científicos, o que não é recomendável.

Uma possível adoção de norma mais simples e de domínio mais amplo significa um ganho na otimização do tempo, dos recursos utilizados ao longo do processo editorial, além de facilitar e simplificar para autores que, de acordo, com o periódico ao qual submetem seus trabalhos, precisam aprender e dominar normas as mais diversas.

No que se refere ao BMPEG. Ciências Humanas sobre o qual trata essa argumentação pela mudança, em última instância representa a internacionalização e a simplificação da norma vigente - a NBR 6023 de elaboração de referências e a NBR 10520 de citações. A verificação de padrões remete para um sistema internacional que esteja em consonância com as principais áreas do periódico Antropologia, Linguística e Arqueologia, e que corresponda aos critérios de qualidades e avalições adotados pelos indexadores. Para esse fim, o estudo analisou estilos normativos bibiográficos em 31 periódicos de caráter internacional em dois indexadores do BMPEG. Ciências Humanas: o Scientific Electronic Library Online (SciELO) e a Red de Revistas Científicas de América Latina y el Caribe, España y Portugal (Redalyc).

Do total de revista analisadas que totalizaram 31 títulos, nove são nacionais e sete internacionais na SciELO e 15 periódicos científicos indexados na Redalyc; todos dedicados à área de Ciências Humanas. A verificação permitiu identificar qual das três normas investigadas: American Psychological Association (APA), Vancouver e Chicago aqui é a mais adotada pelos periódicos dessa área, e dessa forma, indicar um padrão normativo bibliográfico internacional dentre os em uso corrente.

\section{COMUNICAÇÃO CIENTÍFICA}

Em atenção ao conjunto de princípios que regem a comunicação científica na qual se incluem as normas bibliográficas, a publicação de resultados de pesquisas em periódicos especializados se constitui parte intrínseca do processo de produção do conhecimento. Pesquisadores detêm o compromisso de comunicar e de divulgar os estudos que produzem para a sociedade. O BMPEG. Ciências Humanas, como tantos outros periódicos, comunica os resultados científicos por meio canais de formais e informais. O BMPEG. Ciências Humanas, é considerado um canal formal na comunicação científica, logo é possível acessar os artigos pelo site do periódico ${ }^{4}$; pelos seus nove indexadores ${ }^{5}$; e pela plataforma eletrônica, Digital Publishing Platform (ISSUU), entre outros. A revista também divulga os resultados científicos na imprensa especializada e em redes sociais como o Facebook, onde um perfil é alimentado com publicações sobre os artigos publicados e outros itens de interesse ao público leitor do periódico. Meadows (1999, p. 116), confirma que "Entre os cientistas e seu público estão os canais pelos quais eles se comunicam [...]" o autor reitera que a comunicação informal é

\footnotetext{
${ }^{4}$ Disponível em: http://editora.museu-goeldi.br/humanas/. Acesso em: 26 jul. 2018.

${ }^{5}$ Anthropological Index Online; Anthropological Literature; Directory of Open Access Journals (DOAJ); Citas Latinoamericanas en Ciencias Sociales y Humanidades (CLASE); International Bibliography of the Social Sciences (IBSS); Sistema Regional de Información en Línea para Revistas Científicas de América Latina, el Caribe, España y Portugal (Latindex); SCOPUS ELSEVIER; Rede de Revistas Científicas Latinoamericana (Redalyc) e a Scientific Electronic Library Online (SciELO).
} 
efêmera, e que os canais de comunicações formais são os livros e os periódicos (MEADOWS, 1999).

Meadows (1999, p. VII), afirma que "A comunicação situa-se no próprio coração da ciência. É para ela tão vital quanto a própria pesquisa, pois a esta não cabe reivindicar com legitimidade este nome enquanto não houver sido analisada e aceita pelos pares." Ainda para o autor a ciência precisa ser comunicada, e para isso ocorrer é essencial o processo de investigação científica (MEADOWS, 1999).

Para fins desse estudo, tomamos emprestado a Bueno (2010) e a Valerio e Pinheiro (2008), os conceitos que distinguem comunicação de divulgação científica a partir da diferença da forma de apresentação de conteúdos e dos públicos aos quais estão destinadas tais informações. Bueno (2010, p. 5), entende que: “A comunicação científica visa, basicamente, à disseminação de informações especializadas entre os pares, com o intuito de tornar conhecidos, na comunidade científica os avanços obtidos (resultados de pesquisas, relatos de experiências, etc.) [...]", enquanto que a divulgação para o mesmo autor é compreendida como “[...] função primordial: democratizar o acesso ao conhecimento científico e estabelece condições para a chamada alfabetização científica." (BUENO, 2010, p. 5). Valerio e Pinheiro (2008, p. 161), esclarecem que a divulgação científica "[...] visa à comunicação para o público diversificado, fora da comunidade científica.

Nesse sentido, os avanços tecnológicos tendem a provocar grandes mudanças na forma de comunicar a ciência (ALVARENGA, 2003). É o que também afirma Simeão (2006), ao revelar a existência de duas formas de se comunicar a ciência seja pelo modelo intensivo ou extensivo: o primeiro adota o formato tradicional - o impresso, enquanto que o modelo extensivo utiliza o suporte digital. Para Simeão e Miranda (2003, p. 21), a definição de comunicação extensiva consiste na: "[...] interação de emissores e receptores com uma lógica hipertextual, pontual e objetiva em suas metas, mas efêmera, sem estoques e em constante mutação." Sendo assim, as flexibilidades dos avanços tecnológicos exigem "[...] uma reorganização dos conceitos relativos aos processos de comunicação da informação registrada, e seus estatutos.” (MIRANDA; SIMEÃO, 2004, p. 36). E, com essa nova forma de comunicar os resultados científicos, é que as referências possuem um papel importante o de recuperar as informações citadas no decorrer do texto; evitar o plágio/autoplágio na pesquisa científica; e localizar as fontes consultadas.

\section{METODOLOGIA}

Este estudo, de caráter quali-quantitativo, averiguou três estilos normativos de uso internacional: American Psychological Association (APA), Vancouver e Chicago. A primeira norma, denominada APA, segue convenções estabelecidas pela American Psychological Association, e é reconhecida como norma voltada para as grandes áreas das Ciências Sociais e das Ciências Humanas especialmente para as áreas de Psicologia e Antropologia (UNIVERSIDADE DE AVEIRO, 2010). Já o estilo Vancouver é usado para a normalização de referências bibliográficas nas áreas Ciências da Saúde e Ciências Exatas (UNIVERSIDADE DE AVEIRO, 2010). Finalmente, a norma conhecida como Chicago e também como por Turabian Style ${ }^{6}$, está voltada para a área de Ciências Humanas principalmente para disciplinas como a Literatura, a História e as Artes), Ciências Sociais e Ciências Naturais

\footnotetext{
${ }^{6}$ A Universidade de Chicago([2018?]) afirma que o "A Manual for writers of researach - also known as "Turabian" - is the fold standard for college and graduate students in virtually all academic áreas. An introduction to Chicago-stylke formatting and Citation generation, the manual aids students in clear writing, citing, and research practice. At the heart of Turabian is the idea that, no matter the format, the foundations of good research remain the same: to do it carefully, presente it clearly and accurately, and follow academic standards for Citation, style and format. Disponível em: https://wwwchicagomanualofstyle.org/turabian.html.
} 
(UNIVERSIDADE DE AVEIRO, 2010). A revisão da norma visa simplificar o padrão normativo tanto para autores como para editores com vistas à utilização de um formato mais corrente internacionalmente.

Dos nove indexadores que credenciam o BMPEG. Ciências Humanas, foram analisados periódicos em dois indexadores: na SciELO e na Redalyc. Neles analisamos os periódicos científicos voltados exclusivamente para a área de Ciências Humanas, conforme o escopo do BMPEG. Ciências Humanas. Para se identificar a norma padrão adotada pelas revistas foram consultadas as instruções para os autores.

Para corroborar ainda mais a necessidade de alteração da norma BMPEG. Ciências Humanas foram entrevistadas a atual editora científica do periódico a jornalista Jimena Felipe Beltrão e a editora associada e antropóloga Claudia Leonor López Garcés.

Com o intuito de averiguar a norma adotada pelos periódicos, foram consultadas as instruções para os autores dos periódicos científicos dedicados às Ciências Humanas com foco nas áreas de Antropologia, Linguística e Arqueologia, na SciELO, e na base de dados Redalyc, ambos indexadores do BMPEG. Ciências Humanas.

$\mathrm{Na}$ Coleção SciELO foram identificados nove periódicos científicos nacionais e sete internacionais (Quadros 1 e 2). Para fins de diferenciação, os estilos normativos bibliográficos adotados pelos periódicos científicos investigados foram identificados por meio de cores, onde a cor verde representa o estilo APA (11 títulos); o azul, a ABNT (4 títulos); o amarelo, estilo Chicago (1 título); e o laranja, a norma Harvard (1 título).

Quadro 1- Títulos nacionais indexados na SciELO

\begin{tabular}{|l|l|}
\hline \multirow{5}{*}{ Brasil } & $\begin{array}{l}\text {-DELTA: Documentação de Estudos em Lingüística Teórica e } \\
\text { Aplicada }\end{array}$ \\
\cline { 2 - 3 } & -Mana: Estudos de Antropologia Social \\
\cline { 2 - 3 } & -Tempo Social: Revista de Sociologia da USP \\
\cline { 2 - 2 } & -Trabalhos em Linguística Aplicada \\
\cline { 2 - 2 } & -ALFA: Revista de Linguística \\
\cline { 2 - 2 } & -Boletim do Museu Paraense Emílio Goeldi. Ciências Humanas \\
\cline { 2 - 2 } & -Horizontes Antropológicos \\
\cline { 2 - 2 } & -Revista Brasileira de Linguística Aplicada \\
\cline { 2 - 2 } & -Revista de Antropologia \\
\hline
\end{tabular}

Quadro 2 - Títulos internacionais indexados na SciELO

\begin{tabular}{|c|l|}
\hline \multirow{2}{*}{ Argentina } & -Argos \\
\cline { 2 - 2 } & -Comechingonia: Revista de Arqueologia \\
\cline { 2 - 2 } & -Intersecciones en Antropología \\
\hline \multirow{2}{*}{ Peru } & -Anthropologica: Departamento de Ciencias Sociales \\
\cline { 2 - 2 } & -Lexis \\
\hline Uruguai & -Revista Uruguaya de Antropología y Etnografía \\
\hline Venezuela & -Boletín de Lingüística \\
\hline
\end{tabular}

$\mathrm{Na}$ Redalyc, foram realizadas buscas de revistas científicas por país, com posterior aplicação de filtro para a busca por disciplina, em consonância com as três principais áreas de BMPEG. Ciências Humanas como indica o (Quadro 3). 
Quadro 3- Títulos internacionais indexados na Redalyc

\begin{tabular}{|c|l|}
\hline \multirow{4}{*}{ Brasil } & -Boletim do Museu Paraense Emílio Goeldi. Ciências Humanas \\
\cline { 2 - 2 } México & -VIBRANT: Vibrant Virtual Brasilian Anthropology \\
\hline \multirow{4}{*}{ Colombia } & -Alteridades \\
\cline { 2 - 2 } & -Cuicuilco Revista de Ciencias Antropológicas \\
\cline { 2 - 2 } & -Antípoda: revista de Antropología y Arqueologia \\
\cline { 2 - 2 } & -Revista Colombiana de Antropología \\
\cline { 2 - 2 } Chile & -Universitas Humanística \\
\hline \multirow{3}{*}{ Argentina } & -Chungara: revista de Antropología Chilena \\
\cline { 2 - 2 } & -Andes \\
\cline { 2 - 2 } & -Cuadernos de Antropología Social \\
\cline { 2 - 2 } & -Revista Argentina de Antropología Biológica \\
\cline { 2 - 2 } & -Runa \\
\hline Venezuela & -Boletín Antropológico \\
\hline Perú & -Anthropologica del Departamento de Ciencias Sociales \\
\hline
\end{tabular}

É importante esclarecer que o estudo analisou, além de títulos de origem brasileira, outros de origem latino-americana e de origem norte-americana, com o objetivo de detectar a norma mais utilizada pelos periódicos afins ao BMPEG. Ciências Humanas. Em razão da ausência de revistas científicas de origem norte-americana na SciELO, consultou-se o Portal de Periódico da CAPES, onde foi encontrada para a área de Ciências Humanas a Cultural Anthropology.

\section{RESULTADOS}

A indexação de periódicos científicos em bases internacionais reconhecidas representa o reconhecimento e o credenciamento à internacionalização do conhecimento científico, que potencializa o aumento de citações de artigos. Os indexadores que credenciam o BMPEG. Ciências Humanas não impõem ou estabelecem padrões de cunho nacional ou internacional na elaboração de referências e de citações, apenas informam a importância em manter o rigor da norma adotada. Nesse sentido a SciELO, afirma:

A estruturação dos textos é dependente das áreas temáticas e tipos de documentos. Os periódicos devem preferencialmente seguir padrões e práticas mais comuns nas respectivas áreas temáticas [...] para as citações e referências bibliográficas recomenda-se a adoção fiel de normas estabelecidas formalmente como padrões nacional e/ou internacional e mais utilizadas internacionalmente na área temática do periódico [...]. (SCIENTIFIC ELECTRONIC LIBRARY ONLINE, 2017, p. 18-19).

Nessa perspectiva Santos (2010, p.113), corrobora:

[...] a normalização das referências possibilita a automatização de processos de identificação de referências, favorece o desenvolvimento de serviços como exportação de referências bibliográficas para gerenciadores de referências, criação de links com outras fontes de informação, além de permitir a troca de informação entre bases de dados de acesso livre, o que facilita o acesso e a disseminação da informação na web. 
Dos nove periódicos nacionais consultados na Coleção SciELO, quatro adotam a ABNT (NBR 6023 normas de elaboração de referências e a NBR 10520 de citação). Enquanto que, quatro revistas adotam o estilo APA e um periódico utiliza o estilo Chicago. A pesquisa ainda identificou na SciELO sete periódicos científicos internacionais os quais todos adotam o estilo APA em suas publicações. A revista Cultural Anthropology utiliza o estilo Chicago, sistema autor data, no qual a elaboração de referências inclui o sobrenome do autor, seguido da data de publicação do documento.

Já na Redalyc foram encontrados 15 periódicos científicos para os quais foram detectados quatro estilos normativos: APA, Chicago, ABNT e Harvard, dos quais, 14 títulos adotam estilos internacionais, e um utiliza o padrão nacional brasileiro.

Os resultados obtidos na Redalyc demonstram que seis usam a APA e sete, o padrão Chicago. Somente uma revista adota o padrão Harvard (apesar dessa norma não estar entre os três estilos aqui investigados ela é adotada por um dos periódicos indexados na Redalyc), e apenas o BMPEG. Ciências Humanas utiliza a ABNT na normalização dos artigos científicos.

A maioria das revistas examinadas adotam normas de caráter internacional na publicação de artigos científicos. Santos (2010, p. 47) afirma que no "[...] âmbito da normalização científica, alguns estudos avaliaram o grau de ajustes dos periódicos às normas internacionais de apresentação de publicações científicas como elemento importante no êxito da comunicação [...].”A autora aponta ainda a relevância da normalização quando diz que ela contribui no "[...] processo de transferência da informação científica, uma vez que facilita o trabalho dos agentes do circuito de comunicação primária (autores, editores, impressores e leitores) [...].” (SANTOS, 2010, p. 47). O levantamento dos títulos de alcance nacional e internacional dedicados às Ciências Humanas, nos permitiu sugerir alteração ${ }^{7}$ das Normas Brasileiras: a 6023 de elaboração de referências e a 10520 de citações pela norma internacional American Psychological Association (APA) sistema autor data, por ser a mais utilizada pelas revistas da área segundo demonstrado. Esse padrão normativo tem outro aspecto favorável: o acesso gratuito aos dois tutoriais: The Basics of APA Style and Publication Manual of the American Psychological Association ${ }^{8}$.

Para ratificar ainda mais a necessidade de mudança na normalização dos artigos submetidos e aceitos pelo BMPEG. Ciências Humanas foi identificada entre os periódicos científicos nacionais e internacionais investigados na Coleção SciELO a existência de um serviço chamado "Como citar este artigo" nele destacam-se três normas: International Organization for Standardization (ISO), Associação Brasileira de Normas Técnicas (ABNT) e a American Psychological Association (APA), conforme a (Figura 1).

\footnotetext{
${ }^{7}$ Em documento analisado sob a guarda do Arquivo Guilherme de La Penha do Museu Paraense Emílio Goeldi (MPEG) intitulado "Agenda da reunião da comissão de editoração do Museu Paraense Emílio Goeldi”, discutiuse a alteração da norma de elaboração de referências bibliográficas para o uso da ABNT, e não mais as do IBICT por motivos principalmente econômicos (MUSEU PARAENSE EMÍLIO GOELDI, 1987).

${ }^{8}$ The Publication Manual of the American Psychological Association (6th) "[...] outlines the key elements to include when referencing electronic sources, with an emphasis on using the digital object identifier (DOI) to locate web-based information reliably. Most important, it provides a wealth of examples for readers to model for everything from online journal articles to supplemental data sets and measurement instruments to books, videos, apps, websites, podcasts, blog posts, and social media. Approximately 70 examples are provided for readers to consider as they learn how to create reliable references for electronic sources." (American Psychological Association, 2018).
} 
Figura 1- Exemplos de citações disponíveis na Coleção SciELO

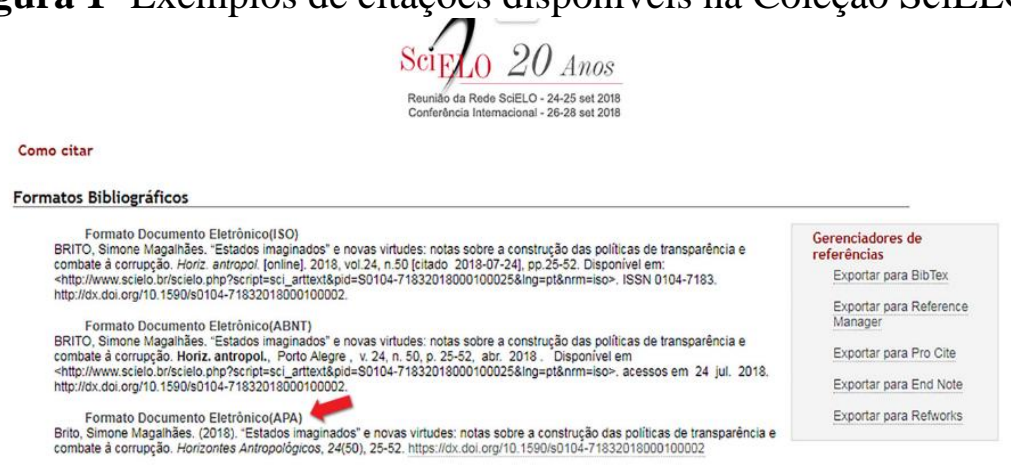

Fonte: Scientific Electronic Library Online (2018).

Observa-se entre as três normas utilizadas na (Figura 1) que o estilo APA é o mais simples de se elaborar, em relação as normas da ISO e da ABNT exemplificadas pela plataforma. Autores como Hoppen, Magnus e Vanz (2018), recomendam que, apesar do Brasil possuir uma norma própria para a elaboração de referências e de citações, criados pela ABNT os "[...] pesquisadores e editores da área biomédica, ciências sociais aplicadas e humanas precisam utilizar as normas já consagradas internacionalmente neste meio - Vancouver e APA." (HOPPEN; MAGNUS; VANZ, 2018, p. 44).

\section{CONSIDERAÇÕES E RECOMENDAÇÃO FINAL}

O BMPEG. Ciências Humanas tem reconhecido papel no ambiente científico por sua longevidade, por sua dedicação e foco sobre temáticas amazônicas. De circulação nacional e internacional, a revista é uma referência nas áreas da Antropologia, da Arqueologia e da Linguística.

Ao analisar as normas adotadas pelos periódicos científicos nacionais e internacionais na Coleção SciELO e no Portal de Periódicos da Capes, é possível sugerir para a normalização dos artigos científicos BMPEG. Ciências Humanas o estilo American Psychological Association (APA) o sistema autor data ${ }^{9}$. Por ser considerado um sistema simples, e de acordo com o que é aqui demonstrado, a maioria das revistas investigadas, na área de Ciências Humanas adotam esse sistema na normalização das referências e das citações. Hoppen, Magnus e Vanz (2018, p. 56), afirmam que "A ampliação das publicações científicas brasileiras no cenário mundial através da indexação em grandes bases de dados impõe a tarefa de cuidar dos aspectos de conteúdo [...], [e] na adequação às normas internacionais."

É o que corrobora a jornalista e atual editora científica do BMPEG. Ciências Humanas, Jimena Felipe Beltrão, quando questionada a respeito do uso da ABNT referentes à elaboração de referências (NBR 6023/2002) e a de citações (NBR 10520/2002):

Considerando que a ABNT é uma norma de uso absolutamente brasileiro, é necessário verificar sua permanência em periódicos como o Boletim de caráter internacional- publicado em quatro línguas e de circulação externa ao país. Entendo que o Boletim deveria, no futuro próximo, considerar normas de uso mais corrente, mais utilizadas internacionalmente. Identificar um modelo simples que mantenha a possibilidade de identificação imediata da referência, inclusive com o uso de DOI e de URL que facilita o acesso imediato pelo leitor, deve se constituir meta. É conhecido que há opções de normas já consagradas e que podem, perfeitamente, ser incorporadas à edição ${ }^{10}$

\footnotetext{
${ }^{9}$ Ver Apêndice A- Exemplos de referências no estilo APA.

${ }^{10}$ BELTRÃO, Jimena Felipe. Entrevista concedida a Taíse Silva. Belém, 22 maio 2018.
} 
A antropóloga Claudia Leonor López Garcés, editora associada para Antropologia no BMPEG. Ciências Humanas, em entrevista voltada para os estudos sobre a revista opina: "As revistas adotam normas e critérios distintos. Para realizar uma possível troca, é importante que haja o estudo das normas: de caráter nacional e internacional, tendo em vista propor a melhor norma que possa atender ao periódico." 11

Analisadas as normas, indica-se que a sua simplificação pode ser auxiliar em práticas de Ciência aberta, uma tendência natural no campo científico, rompendo barreiras para democratizar o conhecimento dentre todos os que dele necessitam tanto no âmbito da pesquisa científica como no cotidiano da sociedade que se vale da Ciência para alcançar melhores níveis de qualidade da vida humana.

\section{AGRADECIMENTOS}

Ao Conselho Nacional de Desenvolvimento Científico e Tecnológico (CNPq) e ao Programa de Capacitação Institucional (PCI) do Museu Paraense Emílio Goeldi -MPEG/MCTI pela concessão de bolsa a uma das autoras deste artigo.

\section{REFERÊNCIAS}

ALVARENGA, Lídia. Representação do conhecimento na perspectiva da Ciência da Informação em tempo e espaço digitais. Encontros Bibli: revista eletrônica de biblioteconomia e ciência da informação. Florianópolis, Santa Catarina, v. 8, n. 15, p. 18-40, 2003. DOI: https://doi.org/10.5007/1518-2924.2003v8n15p18.

AMERICAN PSYCHOLOGICAL ASSOCIATION. APA style guide to electronic references, 2018. Disponível em: http://www.apastyle.org/products/4210512.aspx. Acesso em: 26 July 2018.

ASSOCIAÇÃO BRASILEIRA DE NORMAS TÉNICAS. ABNT NBR 6023: informação e documentação: referências: elaboração. Rio de Janeiro: ABNT, 2018.

ASSOCIAÇÃO BRASILEIRA DE NORMAS TÉNICAS. ABNT NBR 6023: informação e documentação: referências: elaboração. Rio de Janeiro: ABNT, 2002.

BOHANNON, John. Who's afraid of peer review? Science, Washington, v. 342, n. 6141, p. 60-65, 2013. DOI: 10.1126/science.342.6154.60.

BOLETIM DO MUSEU PARAENSE EMÍLIO GOELDI. CIÊNCIAS HUMANAS. Plano de desenvolvimento editorial: situação atual e evolução até o final de 2021. Belém, 2018. 6 p.

BUENO, Wilson Costa. Comunicação científica e divulgação científica: aproximações e rupturas conceituais. Informação e Informação, Londrina, v. 15, p. 1-12, 2010. Edição especial. DOI: http://dx.doi.org/10.5433/1981-8920.2010v15n1espp1.

HOPPEN, Natascha Helena Franz; MAGNUS, Ana Paula Medeiros; VANZ, Samile Andréa de Souza. Referências de atos normativos brasileiros na comunicação científica internacional:

${ }^{11}$ GARCÉS, Claudia Leonor López. Entrevista concedida a Taíse Silva. Belém, 22 maio 2018. 
estilos Vancouver e APA. Prisma com, Portugal, n. 36, p. 41-58, 2018. DOI: $10.21747 / 16463153 / 36 a 3$.

MAIMONE, Giovana; TÁLAMO, Maria de Fátima. A atuação do bibliotecário no processo de editoração de periódicos científicos. Revista ACB, Santa Catarina, Florianópolis, v. 13, n. 2, p. 301-321, jul./dez. 2008.

MEADOWS, Arthur Jack. A comunicação científica. Rio de Janeiro: Briquet de Lemos, 1999.

MEDEIROS, Graziela Martins de; FACHIN, Gleisy Regina Bóries; RADOS, Gregório Jean Varvakis. Padronização de periódicos científicos on-line da área de biblioteconomia e Ciência da Informação: adequação as normas ISO. Revista ACB, Santa Catarina, Florianópolis, v. 13, n. 2, p. 415-438, jul./dez. 2008.

MIRANDA, Antonio; SIMEÃO, Elmira. Transferência de informação e transferência de tecnologia no modelo de comunicação extensiva: a BABEL.com. Informació, Cultura y Sociedad, Buenos Aires, n. 10, p. 27-40, 2004.

MUSEU PARAENSE EMÍlIO GOELDI. Arquivo Guilherme de La Penha. Agenda da reunião da comissão de editoração do Museu Paraense Emílio Goeldi. Belém, 1987.

RODRIGUES, Eloy. Acesso livre ao conhecimento: a mudança do sistema de comunicação da ciência e os profissionais de informação. Cadernos Bad: revista da associação portuguesa de bibliotecários, arquivistas e documentalistas, Portugal, n. 1, p. 24-35, 2004.

SANTOS, Solange Maria dos. Perfil dos periódicos científicos de ciências Sociais e de Humanidades: mapeamento das características extrínsecas. 2010. 176f. Dissertação (Mestrado em Ciência da Informação) - Universidade de são Paulo, São Paulo, 2010.

SANTA ANA, Jorge. Normalização bibliográfica no âmbito da transferência da informação: de um fazer técnico a uma atuação humanista. Revista ACB, Santa Catarina, Florianópolis, v. 22, n. 1, p. 61-76, dez./mar. 2017.

SABADINI, Aparecida Angélica Zoqui Paulovic; SAMPAIO, Maria Imaculada Cardoso; NASCIMENTO, Maria Marta. Preparando um periódico científico. In: SABADINI, Aparecida Angélica Zoqui Paulovic; SAMPAIO, Maria Imaculada Cardoso; KOLLER, Sílvia Helena (org.). Publicar em Psicologia um enfoque para a revista científica. São Paulo: Associação Brasileira de Editores Científicos de Psicologia/Instituto de Psicologia da Universidade de São Paulo, 2009. p. 35-73.

SIMEÃO, Elmira. O modelo de comunicação extensiva e as implicações no contexto da comunicação científica: metodologia para mensuração de indicadores do formato eletrônico em rede. In: CONFERÊNCIA IBEROAMERICANA DE PUBLICAÇÕES ELETRÔNICAS NO CONTEXTO DA COMUNICAÇÃO CIENTÍFICA, 1., 2006, Brasília. Anais [...]. Brasília, 2006.

; MIRANDA, Antonio. Comunicação extensiva e a linguagem plástica dos documentos em rede. In: RODRIGUES, Georget Medleg; LOPES, Ilza Leite (org.). Organização e representação do conhecimento na perspectiva da Ciência da 
Informação. 1. ed. Brasília: Thesaurus, 2003. p. 179-209. (Estudos avançados em Ciência da Informação, v. 2).

SCIENTIFIC ELECTRONIC LIBRARY ONLINE (SciELO). Critérios, política e procedimentos para admissão e a permanência de periódicos científicos na Coleção SciELO Brasil. São Paulo, 2017. 31 p. Disponível em:

http://www.scielo.br/avaliacao/Criterios_SciELO_Brasil_versao_revisada_atualizada_outubro _20171206.pdf. Acesso em: 19 jul. 2018.

STUMPF, Ida. Avaliação pelos pares nas revistas de comunicação: visão dos editores, autores e avaliadores. Perspectiva em Ciência da Informação, Belo Horizonte, v. 13, n. 1, p. 18-32, jan./abr. 2008.

THE ROYAL SOCIETY. Science as an open. London: The Royal Society, 2012. Disponível em: https://royalsociety.org/topics-policy/projects/science-public-enterprise/Report/. Acesso em: 23 out. 2018.

UNIVERSIDADE DE AVEIRO. Serviços de Biblioteca, Informação Documental e Museologia. Referências bibliográficas: manual de normas e estilos. Aveiro, Portugal: Bibliotecas da Universidade de Aveiro, 2010.

UNIVERSIDADE OF CHICAGO. Turabian: a manual for writers. Chicago, ([2018?]). Disponível em: https://www.chicagomanualofstyle.org/turabian.html. Acesso em: 31 July 2018.

VALERIO, Palmira Moriconi; PINHEIRO, Lena Vania Ribeiro. Da comunicação científica à divulgação. Transinformação, Campinas, v. 20, n. 2, p. 159-169,2008. DOI:

http://dx.doi.org/10.1590/S0103-37862008000200004.

\section{APÊNDICE A- Exemplos de referências no estilo APA}

\section{Livro:}

Veríssimo, J. (1906). A educação nacional. (2a ed.). Rio de Janeiro: Livraria Francisco Alves.

Wieczorek, A., Rosendahl, W., \& Schlothauer, A. (Orgs.) (2012). Der Kult um Kopf und Schädel. Heidelberg: Verlag Regionalkultur.

Série/Coleção:

Goeldi, E. (1900). Escavações arqueológicas em 1895: Executadas pelo Museu Paraense no litoral da Guiana Brasileira entre Oiapoque e Amazonas. (Memórias do Museu Goeldi, n. 1). Belém: Museu Paraense de História Natural e Ethonografia.

Carneiro da Cunha, M. (1992). Política indigenista no século XIX. In M. Carneiro da Cunha (Orgs.), História dos índios no Brasil (pp. 133-154). São Paulo: Companhia das letras.

Artigo de periódico: 
Gurgel, C. (1997). Reforma do Estado e segurança pública. Política e Administração, 3(2), 15 21.

\section{Artigo de periódico sem DOI:}

Tersis, N., \& Carter-Thomas, Shirley. (2005). Investigating syntax and pragmatics: Word order and transitivity in Tunumiisut. International Journal of American Linguistics, 71(4), 473500 .

\section{Artigo de periódico em meio eletrônico com DOI:}

Velthem, L. H. V. (2012). O objeto etnográfico é irredutível? Pistas sobre novos sentidos e análises. Boletim do Museu Paraense Emílio Goeldi. Ciências Humanas, 7(1), 51-66. Recuperado de http://www.scielo.br/scielo.php?script=sci_arttext\&pid=S198181222012000100005\&lng=pt\&nrm=iso\&tlng=pt.doi: http://dx.doi.org/10.1590/S1981$\underline{81222012000100005}$

Artigo e/ou matéria de jornal:

Naves, P. (1999, junho 28). Lagos andinos dão banho de beleza. Folha de S. Paulo, Turismo, Caderno 8, p. 13.

Artigo e/ou matéria de jornal em meio eletrônico:

Silva, I. G. (1998, setembro 19). Pena de morte para o nascituro. O estado de S. Paulo. Recuperado de http://www.portaldafamilia.org/artigos/artigo225.shtml

\section{Trabalho apresentado em evento:}

Brayner, A. R. A., \& Medeiros, C. (1994). Incorporação do tempo em SGBD orientado a objetos. In Simpósio Brasileiro de Banco de Dados, Anais 2009 (pp. 16-29). São Paulo.

\section{Trabalho apresentado em evento em meio eletrônico:}

Silva, R. N., \& Oliveira, O. (1996 janeiro). Os limites pedagógicos do paradigma da qualidade total na educação. Anais eletrônicos do Congresso de Iniciação Científica da UFPe, Recife, PE, Brasil, 4. Recuperado de https://www.ufpe.br/propesq/anais/educ/ce04.htm

Mensagens de Internet: lista de discussão eletrônica e outras comunidades online:

Boletim do Museu Paraense Emílio Goeldi. Ciências Humanas (2018, 16 julho). Boletim de Ciências Humanas do Museu Paraense Emílio Goeldi discute patrimônio indígena [Facebook]. Recuperado de https://www.facebook.com/boletimgoeldiCH/

Blog SciElo em Perspectiva Humanas (2018, junho 13). Cultura ancestral para entender a Amazônia de ontem e de hoje [Blog]. Recuperado de http://humanas.blog.scielo.org/blog/2018/06/13/cultura-ancestral-para-entender-aamazonia-de-ontem-e-de-hoje/

Documento jurídico: Resolução $n .^{\circ}$ 17, de junho de 1991. Coleção de Leis da República Federativa do Brasil. Recuperado de http://www2.camara.leg.br/legin/fed/ressen/1991/resolucao-17-14-junho-1991-480998publicacaooriginal-1-pl.html

Documento jurídico: Medida Provisória n $^{\circ}$ 1.569-9, de 11 de dezembro de 1997. Estabelece multa em operações de importação, e dá outras providências. Recuperado 
http://www2.camara.leg.br/legin/fed/medpro/1997/medidaprovisoria-1569-9-11dezembro-1997-377059-publicacaooriginal-1-pe.html

Trabalhos acadêmicos (teses, dissertações e monografias):

Benchimol, A. (2015). Resgate e ressignificação da pesquisa no Museu Paraense Emílio Goeldi: Presença e permanência de cientistas estrangeiros (1894-1914) na produção científica de autores atuais (1991-2010) (Tese de doutorado). Instituto Brasileiro de Informação em Ciência e Tecnologia, Universidade Federal do Rio de Janeiro, Rio de Janeiro, RJ, Brasil.

Moore, D. (1984). Syntax of the language of the Gavião Indians of Rondônia, Brazil. (Tese de doutorado). University of New York, Nova York. 